\title{
Comments on the Cerebral Edema After CPR: A Therapeutic Target Following Cardiac Arrest?
}

\author{
Xiaohong Wang ${ }^{1} \cdot$ Yan $\mathrm{Li}^{2} \cdot$ Qing Sun ${ }^{1} \cdot$ Qiang $\mathrm{Sun}^{3}$ \\ Published online: 5 March 2018 \\ (C) Springer Science+Business Media, LLC, part of Springer Nature \& Neurocritical Care Society 2018
}

Dear Editor,

A review recently published by Dr. Hayman et al. [1] on October 2017 issue of Neurocritical Care has summarized the cerebral edema's role in neurological outcome following cardiac arrest (CA). However, we are sorry to notice that the authors have left hyperbaric oxygen therapy (HBOT) out of the "management of cerebral edema: clinical approaches" section of their cogitative review, as we believe this is a very effective way of alleviating cerebral edema caused by CA.

Hyperbaric oxygen has long been used clinically for treating multiple indications. As a therapy breathing $100 \%$ oxygen in a pressurized room, early induction of HBOT has been proven neuroprotective by reducing cerebral edema in both animals [2] and humans [3]. Although there are doubts concerning oxygen given its toxicity in excess, the application of multiple therapeutic interventions like hyperbaric oxygen (as a primary factor) in combination with free radical scavengers or inhibitors of free radicalgenerating enzymes would counteract these possible side effects while simultaneously maximizing the HBOT's treating advantages.

The mechanism of HBOT clinically managing cerebral edema and improving neurological outcome after cardiopulmonary resuscitation (CPR) involves promoting the growth of neurons, protecting blood brain barriers integrity, lessening cell apoptosis, and limiting the inflammatory response [4]. Interestingly, HBOT has been reported as an effective method of lowering brain water content in the

Qiang Sun

sunqiang83@gmail.com

1 Department of Hyperbaric Medicine, No. 401 Hospital of the People's Liberation Army, Qingdao 266071, China

2 State Food and Drug Administration of the West Coast New Area, Qingdao 266555, China

3 Department of Hyperbaric Medicine, PLA Navy General Hospital, Beijing 100048, China literature, just like the hyperosmotic fluid does in intracerebral pressure control after CA [5]. Clinically speaking, given that a standardized protocol for HBOT (e.g., pressure, daily duration, total times, and period of treatment) for treating patients with cerebral edema post-CPR onset is highly controversial, more carefully designed, doubleblind, random, placebo-controlled trials should and must be conducted for the sake of evidence-based medicine in the future.

In conclusion, HBOT, despite possibly not as a primary therapeutic choice to treat cerebral edema post-CPR onset, should be at least introduced for academic community to discuss in this guiding review.

\section{Compliance with Ethical Standards}

Conflict of interest The authors declare that they have no conflict of interest.

\section{References}

1. Hayman EG, Patel AP, Kimberly WT, Sheth KN, Simard JM. Cerebral edema after cardiopulmonary resuscitation: a therapeutic target following cardiac arrest? Neurocrit Care. 2017. https://doi. org/10.1007/s12028-017-0474-8.

2. Rosenthal RE, Silbergleit R, Hof PR, Haywood Y, Fiskum G. Hyperbaric oxygen reduces neuronal death and improves neurological outcome after canine cardiac arrest. Stroke J Cereb Circ. 2003;34:1311-6.

3. Hu W, Chao Y, Geng X. Neuroprotective strategies for patients with acute myocardial infarction combined with hypoxic ischemic encephalopathy in the ICU. Hell J Cardiol. 2017. https://doi.org/ 10.1016/j.hjc.2016.12.006.

4. Matchett GA, Martin RD, Zhang JH. Hyperbaric oxygen therapy and cerebral ischemia: neuroprotective mechanisms. Neurol Res. 2009;31:114-21.

5. Zhang Y, Yang Y, Tang H, et al. Hyperbaric oxygen therapy ameliorates local brain metabolism, brain edema and inflammatory response in a blast-induced traumatic brain injury model in rabbits. Neurochem Res. 2014;39:950-60. 